\title{
The origin of radio relics in galaxy clusters: a combined $\gamma$ and radio wavelength analysis
}

\section{Claudio Moises Paulo*}

School of Physics, University of the Witwatersrand, Johannesburg, Wits 2050, South Africa

Departamento de Física, Universidade Eduardo Mondlane, Po Box 1569, Maputo, Moçambique

E-mail: Claudio.Paulo@students.wits.ac.za

\section{Sergio Colafrancesco}

School of Physics, University of the Witwatersrand, Johannesburg, Wits 2050, South Africa

E-mail: sergio.colafrancescodwits.ac.za

\section{Paolo Marchegiani}

School of Physics, University of the Witwatersrand, Johannesburg, Wits 2050, South Africa

E-mail: Paolo.Marchegiani@wits.ac.za

Radio relics are diffuse radio sources which are detected usually at the outskirts of galaxy clusters. They provide important information about the origin of non-thermal processes taking place in the cluster atmospheres. At the time of writing this paper, 78 relics are known, while currently available models predict that a much larger number of these sources should exist.

We discuss in this paper the constraints on the combined radio and $\gamma$-ray emission from these structures in galaxy clusters and we propose a new approach to unveil their origin.

Key words. galaxies: clusters: general, galaxies: clusters: intracluster medium, diffuse radio emissions: radio relics, radiation mechanisms: thermal: non-thermal, acceleration of particles, gamma-rays.

4th Annual Conference on High Energy Astrophysics in Southern Africa

25-27 August, 2016

Cape Town, South Africa

\footnotetext{
* Speaker.
} 


\section{Introduction}

Galaxy clusters are the largest gravitationally-bound structures in the universe (28; 44). They are dominated by dark matter $(\sim 80 \%)$ and only a tiny fraction of its mass is in the form of stars in galaxies $(\sim 3-5 \%)(19)$. The remaining $\sim 15-17 \%$ comprises the intra-cluster medium (ICM) (19), i.e. a hot $\left(T \sim 10^{7}-10^{8} \mathrm{~K}\right)$ and tenuous $\left(n \sim 10^{-3} \mathrm{~cm}^{-3}\right)$ ionized plasma that fills the space between galaxies in a cluster (36).

Radio observations of clusters show the presence of diffuse emissions in the form of radio halos, radio mini-halos and radio relics (RRs) depending on their location, morphology and spectral shape (see, e.g., (52; 24) for more details).

RRs, which are the target of our present study, are typically observed toward the cluster periphery at $\sim$ Mpc distances from the cluster centre (44). They are characterized by i) steep radio spectra ( $\alpha \sim 1.0-1.4$, where the flux density spectrum is $S_{v} \propto v^{-\alpha}(8 ; 19)$ ), ii) very low-surface brightness emission $\left(\sim \mu \mathrm{Jy} \operatorname{arcsec}^{-2}\right.$ at $\left.1.4 \mathrm{GHz}\right)(26 ; 29)$, iii) large physical extents $(\sim 1-1.5 \mathrm{Mpc})(44)$, iv) high radio polarization (44), and v) $1.4 \mathrm{GHz}$ radio powers in the range of $10^{23-26} \mathrm{~W} \mathrm{~Hz}^{-1}$ $(19 ; 26 ; 6)$.

Even after decades of studies, the origin of RRs is still unknown. A commonly adopted explanation for their origin is the radio emission from shock (re-)accelerated electrons (see, e.g., $(34 ; 16)$ ) which shows, however, several inconsistencies:

- observations show that the position of RRs does not coincide with the position of X-ray detected shocks in several cases (see, e.g., discussion in (47));

- the Mach number $\mathscr{M}$ at the outer edge of the relic derived from the X-ray temperature difference is significantly lower $(\mathscr{M} \leq 4)$ than the value derived from the radio spectral index assuming a simple diffusive shock acceleration (DSA) theory (see, e.g., $(31 ; 55)$ );

- if the DSA model is correct, we also expect the re-acceleration of cosmic-ray protons in galaxy clusters which should produce intense $\gamma$-ray emission. However, high-energy observations of nearby galaxy clusters have not detected any diffuse $\gamma$-ray emission $(56 ; 44)$;

- the origin of the relic-halo bridges found in some clusters, e.g. in the Coma cluster, A2255, A2744 (27) and recently in A3667 (17), is neither observationally clear nor theoretically well explained;

- there is often a positional coincidence of powerful radio galaxy jets and radio relics. We can distinguish at least two cases: i) elongated radio relics with a radio galaxy located at one of the edges (e.g. the Bullet cluster, (48)); ii) double arc-like relics with a radio galaxy at the apparent centre of the radio structure (e.g. A3667, (29)).

We point out that the main difficulty in the understanding of the origin of radio relics resides in the low Mach number $(\mathscr{M} \approx 1 \div 4)$ associated with merger shocks at the relic location (11). The acceleration efficiency at these weak shocks is indeed expected to be small and in several cases is in tension with the observational requirements (see, e.g., (16)).

Related to this problem is the lack of detection of cluster $\gamma$-ray emission by Fermi LAT, which puts strong limits on the efficiency of DSA produced at shocks, and this produces a strong tension 
regarding this process as the explanation of radio relics. In fact, while it is known that the process of accelerating electrons from the thermal gas is inefficient compared to the heating produced by these processes (see, e.g., $(60 ; 42)$ ), even the re-acceleration of mildly relativistic electrons is problematic. In fact, the DSA, as well as other acceleration processes related to the presence of shocks and turbulences in galaxy clusters, are recognized to be usually effective both for cosmic rays protons and electrons $(40 ; 14)$; therefore a high level of electron acceleration would also imply an acceleration of relativistic protons, yielding a large amount of gamma rays due to the decay of $\pi^{0}$ produced in hadronic collisions between cosmic-ray protons and the thermal gas nuclei (57).

It is therefore necessary to search for alternative explanations for the origin of relativistic electrons producing the radio relics.

In this paper, we first explore the correlation between the power of a radio relic and the Mach number of the shock in the cluster, to check if the acceleration level of non-thermal electrons is directly connected with the Mach number. After showing that this correlation is not what is expected according to the DSA scenario, we explore the possibility that radio relics are connected to jets of radio galaxies embedded in clusters. We consider here, for the sake of conciseness, the example of two clusters where the relic appears to be associated with a radio-galaxy jet, and discuss how a jet can be correlated with the formation of a radio relic. A wider discussion of this association is presented elsewhere (Colafrancesco et al., in preparation). We finally discuss the possibility to check our hypothesis in the high-energy part of the electromagnetic spectrum.

A flat, vacuum dominated Universe is assumed throughout this paper, with $\Lambda \mathrm{CDM}$ cosmology with $H_{0}=67.3 \mathrm{~km} \mathrm{~s}^{-1} \mathrm{Mpc}^{-1}, \Omega_{\Lambda}=0.68$ and $\Omega_{M}=0.32$.

\section{Correlation between Mach number and relic radio power}

The main problem about the origin of RRs is understanding the source of relativistic electrons that produce radio emission. In the commonly assumed approach (see, e.g., (24)) this is connected to the presence of intra-cluster shocks that are supposed to accelerate particles to high energies through DSA. We notice that such a scenario requires strong shocks with Mach numbers $\mathscr{M}>5$ (see, e.g., (30)). We found that all but three of the 16 RRs analyzed by (29) show Mach numbers, determined from the radio spectral index assuming DSA (see eq.2.1 below), lower than 4, with the exception of the RRs in PLCKG287+32.9 NW (10), CIZAJ2242.8+5301 N (49), and the Toothbrush (54).

In order to accomplish the goals of this study, we evaluated the relation between the radio power at $1.4 \mathrm{GHz}, P_{1.4}$, of relics and Mach number $(\mathscr{M})$. The approach of (3) was adopted to fit a straight line $(y=m x+c)$ to the data set in logarithm scale, therefore corresponding to a power-law relation; we report here the best fit obtained from BCES ${ }^{1}$-bisector estimator described in (3) which treats the variables symmetrically and is recommended for scientific problems where the goal is to estimate relationship between variables and for a comparison with theory (15).

To assess the existence of possible correlations between the selected quantities, the Spearman's rank correlation coefficient (S-rank) was applied $(25 ; 61)$ taking in consideration the significance level: one-tailed/directional with confidence level 0.05 . The null hypothesis for the test in consideration was assumed to be the following: there is no association between variable A and Variable B

\footnotetext{
${ }^{1}$ the Bivariate Correlated Errors and intrinsic Scatter (61).
} 
(25). The null hypothesis is rejected when the value of the coefficient correlation $\rho$ for the sample is higher than or equal to the critical value from the Spearman rank correlation coefficient table, thus considering the findings significant.

The distribution of $\mathscr{M}$ for the RRs in (29) $(\mathrm{N}=15)$ in the $P_{1.4}-\mathscr{M}$ plane is shown in Fig. 1 . The solid red line represents the best-fit obtained for a power-law relation $P_{1.4}=E \cdot(\mathscr{M})^{f}$ and the blue area encompasses the $95 \%$ confidence region (i.e. the region that has $95 \%$ probability to contain the regression line (33)). The best fit obtained from the BCES-bisector has the following param-

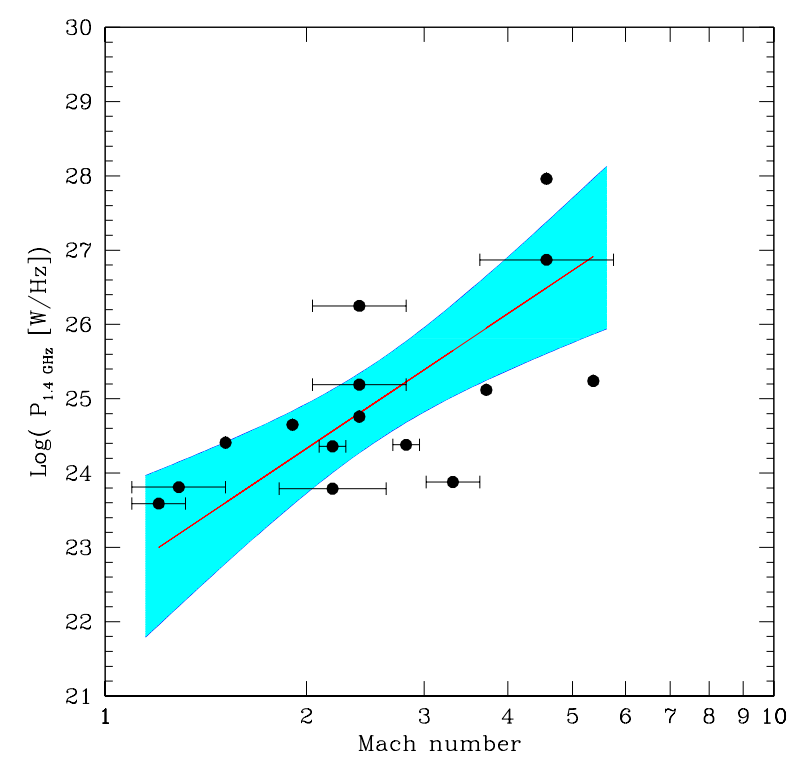

Figure 1: Relic radio power at $1.4 \mathrm{GHz}\left(P_{1.4}\right)$ versus Mach number $(\mathscr{M})$ for the sub-sample of relics with estimated Mach numbers given in (29). The best-fit power law relation $P_{1.4}=E \cdot(\mathscr{M})^{f}$ has a slope $f=6.0 \pm 1.4$ and a normalization of $\log E=22.52 \pm 0.49$ and it is shown by the solid red line. Points without error bars are either lower limits or uncertain estimates (see (29) for details).

eters: the slope is $f=6.0 \pm 1.4$ and the normalization is $\log E=22.52 \pm 0.49$. The correlation coefficient, $\rho$ for this subsample $(\mathrm{N}=15)$ is 0.66 and its significance was tested using the S-rank approach which has a value of 0.446 . Since the value from the $S$-rank approach is smaller than $\rho$, we conclude that $\rho$ is significant and the null hypothesis is rejected for this correlation.

The theoretical correlation expected on the basis of the shock model of (30) indicates that in the strong shock limit $(\mathscr{M}>5)$ a correlation $P_{1.4} \sim$ constant is expected, while in the weak shock limit $(\mathscr{M}<3)$ the radio power is expected to be strongly decreasing with $\mathscr{M}$ (see Fig.6 of (30)).

The observed correlation for the RR sub-sample studied here does not support both the strong and weak shocks limits, because the $P_{1.4}-\mathscr{M}$ relation does not show any change in the slope for the 
different values of $\mathscr{M}$ : for $\mathscr{M}<3$ the observed slope is smaller than the theoretical one, whereas for $\mathscr{M}>3$ we do not observe the expected flattening of the relation. This evidence indicates that the general shock acceleration theory is not consistent with the observed RR radio power distribution and therefore it cannot be considered as a representative model for the origin of radio relics in clusters. This argument seems to strongly contend the DSA scenario for the origin of the relativistic electron population necessary to produce RR emission.

In addition, (35) recently found that the spectral index measured in several relics is not what is expected from the DSA theory, where

$$
\mathscr{M}^{2}=\frac{\alpha+1}{\alpha-1}
$$

(e.g. (7)), and where the Mach number derived from X-ray observations is generally not sufficient to produce the observed values of the radio spectral index, as found also in A2255 (2).

Alternative scenarios for the RR origin involve the presence of a pre-existing population of seed relativistic electrons that might be further revived by magnetic field compression of low- $\mathscr{M}$ shocks.

Another solution that has been proposed is related to the dependence of the shock efficiency on its obliquity, i.e. the angle between the shock normal and the underlying upstream magnetic field. In fact, it has been shown that if the electrons are accelerated only by quasi-perpendicular shocks, and the protons are accelerated only by quasi-parallel shocks, it is possible to reduce the gamma-ray emission predicted by DSA by maintaining a strong radio emission; however, even assuming this strong requirement, in some cases (like the Coma one) the predicted gamma-ray emission is still higher than the Fermi LAT upper limits, therefore the problem is reduced but not entirely solved (59).

In this context, the presence of a population of seed relativistic electrons ejected from early episodes of radio galaxy activity in the form of remnants seems to be a viable possibility for the origin of RRs. Remnants from radio galaxies have in fact been widely observed (see, e.g., (12)) and can also reach quite large sizes and distances from the radio galaxy cores.

We have in fact analyzed the association of active radio galaxies with relics for a sample of 78 RRs (Colafrancesco et al., in preparation) and in the next Section we present this association of active radio galaxies with relics for two representative clusters.

\section{The connection between (active) radio galaxies and relics}

To discuss this scenario we consider two representative cases: i) the Bullet cluster, where an elongated relic is present, and ii) A3667 where a double arc relic is present.

\subsection{The case of 1E0657-55.8}

The galaxy cluster 1E0657-55.8 (usually referred to as the Bullet cluster) at redshift $z=0.296$ is a well-known, extremely hot (average $k T \sim 14.7 \mathrm{keV}$ ) and massive merging cluster with rich gravitational lensing, X-ray and radio data $(48 ; 38)$. X-ray observations revealed a prominent bow shock with $\mathscr{M} \sim 3$ (18). Radio observations of this cluster have reported the presence of a powerful giant radio halo (see e.g. $(47 ; 48 ; 38)$ for more details) and also of a peripheral radio relic (see e.g. 
(48) for details).

The relic of this cluster shows two distinct but physically connected regions (see Fig.2) and has the highest surface brightness among any known cluster relic (48). The region A of the relic (see Fig.2)

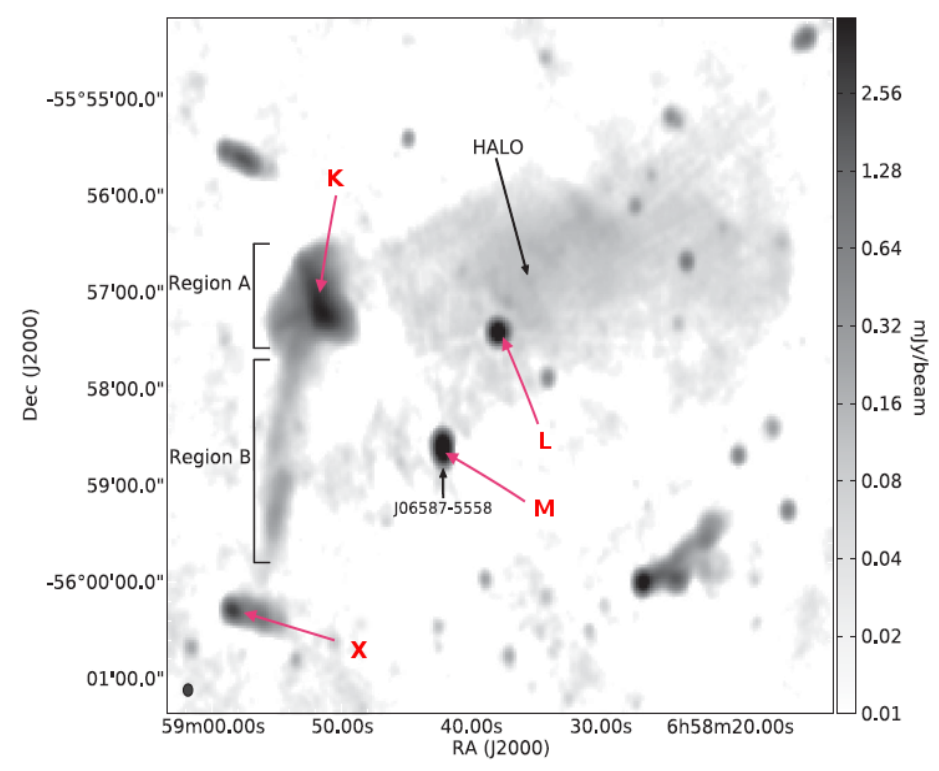

Figure 2: The radio relic of the Bullet cluster as presented in (48) consists of the regions A and B as labelled in the image (see e.g. (48) for details).

has an exceptionally high surface brightness (see Tab.1 for flux information) compared to the faint southern region B: it has been proposed that this brightness enhancement could be due to a large, pre-existing, population of relativistic electrons in this region (48), which were re-accelerated by shocks produced during cluster merger (see, e.g., $(6 ; 55 ; 22 ; 21 ; 11)$ and references therein).

Table 1: Additional information of Bullet cluster radio relics.

\begin{tabular}{cccc}
\hline \hline Source & Int. Flux Density & Int. Flux Density & References \\
Label & $\begin{array}{c}S_{5.5} \mathrm{GHz} \\
(\mathrm{mJy})\end{array}$ & $\begin{array}{c}S_{9} \mathrm{GHz} \\
(\mathrm{mJy})\end{array}$ & \\
& $18.04 \pm 0.40$ & $4.36 \pm 0.15$ & $(38)$ \\
\hline Region A & $0.88 \pm 0.08$ & $0.09 \pm 0.02$ & $(38)$ \\
Region B & 0.96 \\
\hline
\end{tabular}

We note that there is also a very low surface brightness but significant bridge of radio emission between the region B of the radio relic, the radio source J06587-5558, and the south eastern region of the radio halo whose origin is not yet clear (48).

Figure 2 shows numerous radio sources located in the cluster area with some of them embedded within the diffuse radio emission. The positions of those labelled with $\mathrm{L}, \mathrm{M}, \mathrm{K}$, and X suggest that they can be linked to the radio relic properties (see Tab. 2 and Fig. 3) due to their spatial location close to the relic or between the relic and the halo. Therefore it is interesting to study in detail the 
properties of these sources in order to determine if they can really be related to the relic.

Table 2: List of sources spatially connected with the relic in Bullet cluster.

\begin{tabular}{ccc}
\hline $\begin{array}{c}\text { Source } \\
\text { Name }\end{array}$ & $\begin{array}{c}\text { RA } \\
\mathrm{J}(2000) \\
\text { hh mm ss.d }\end{array}$ & $\begin{array}{c}\text { Dec } \\
\mathrm{J}(2000) \\
\text { dd mm ss.d }\end{array}$ \\
\hline K & 065850.0 & -555700.0 \\
L & 065837.9 & -555725.4 \\
M & 065842.1 & -555837.6 \\
X & 065856.7 & -560015.0 \\
\hline
\end{tabular}

It is interesting to note that the source $\mathrm{M}$ is located approximately in the middle of the faint halo-relic bridge, and that this situation is similar to the case of the galaxy NGC 4839 in the Coma cluster, suggesting a possible role of these sources in this kind of radio structures. However, according to $(37 ; 32)$, it is not clear if the source $\mathrm{M}$ is physically associated to the cluster. This source is considered by $(48 ; 37 ; 32)$ to be a gravitational lensed image of a high-redshift radio galaxy or relic. Therefore it is not possible to exclude that the position of $\mathrm{M}$ inside the halo-relic bridge is a coincidence.

A recent analysis of the source $\mathrm{L}$ indicates a relation with a possible Dark Matter sub-halo in the cluster which can be linked to the radio halo emission, or, alternatively, it can be associated to the interaction region between two galaxies (39).

There are not many details available on the sources $\mathrm{X}$ and $\mathrm{K}$ in the literature. However, these two sources look very interesting because they are located close the two edges of the radio relic, suggesting a possible connection with it. We therefore analyzed their spectral energy distribution (SED) in order to derive more information on their nature. 


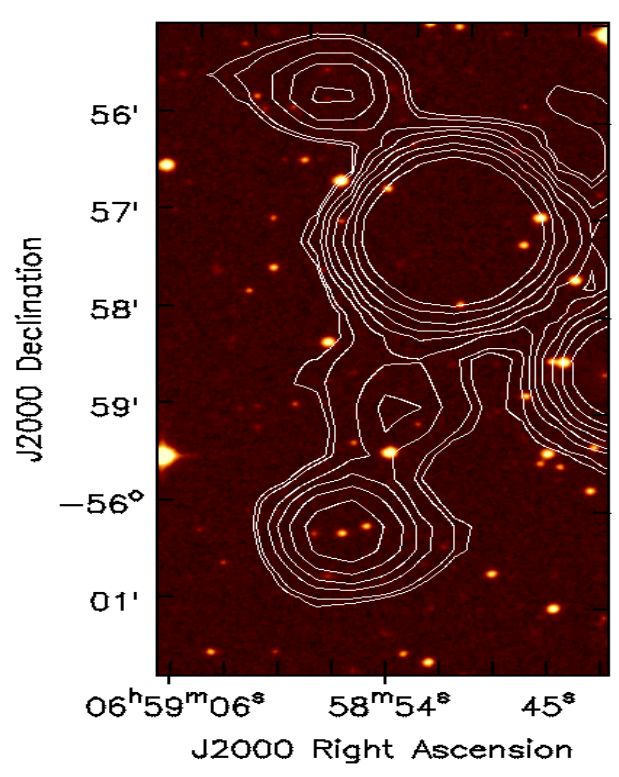

Figure 3: Image of the radio relic emission (overlaid contours of SUMSS from SIFA (The Sydney Institute for Astronomy), at levels (3, 4.2, 8.3, 11.7, 16.5, 23.3, 33, 46.7 mJy beam $^{-1}$ ) superposed onto the DSS-2-red optical image of the cluster showing the association of the relic with optical sources.

We found that there is a SUMSS radio source $(\mathrm{RA}=065856.87$; $\mathrm{DEC}=-560017.28)$ at 2.7 arcsec distance from the coordinates of the source X with a flux of $17.7 \mathrm{mJy}$ at $843 \mathrm{MHz}$ which has also an optical counterpart (within $0.3 \mathrm{arcsec}$ ) in the USNOB1.0 catalog with an $R_{\text {mag }}=16.77$. This source has also recently been observed with ATCA (38), confirming that this galaxy is coincident with the radio source.

Figure 4 shows the SED of this source. We note that the SED of this object shows a non-thermal component in the radio band (see Fig. 4), and a typical galaxy host thermal spectrum in the Infrared/Optical band. To have a more conclusive approach, we need to have optical spectra of this object, to understand: i) if the nuclear activity is strong (e.g. if there are emission lines), and ii) if its redshift can tell us if it is belonging to the cluster or is a background or foreground object. 


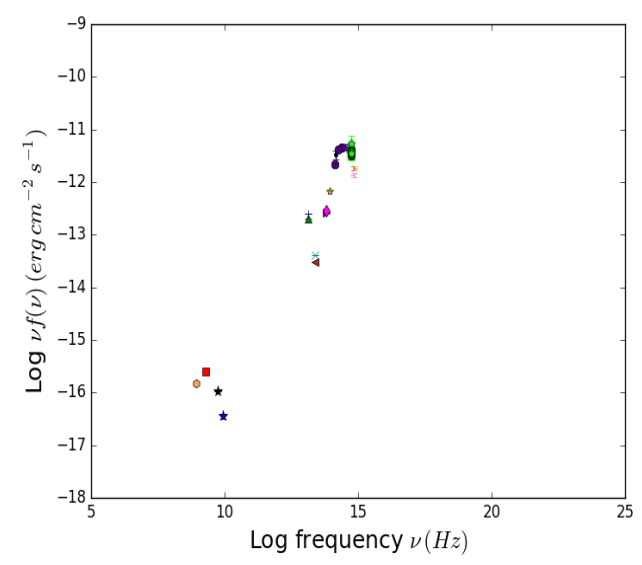

Figure 4: SED of the object associated with the source $X$ in the ATCA radio map.

The SED was produced with data extracted from the SED Builder Tool of the ASDC Sky Explorer. The flux $\left(\log v \cdot f(v)=-15.6 \mathrm{erg} \mathrm{cm}^{-2} \mathrm{~s}^{-1}\right.$ for the source $\left.\mathrm{X}\right)$ shown as a red square represents the ATCA data which was calculated using the contours shown in Fig. 5 in (47). For that, the combination of the contour level $5 \times \sqrt{1,2,4,8, \ldots} \times 25 \mu \mathrm{Jy}_{\text {beam }}{ }^{-1}$, the synthesized FWHM beam $(3.0 \mathrm{arcsec})$ and the average frequency range of $2.1 \mathrm{GHz}$ were used. These ATCA data position according to the SED of non-thermal sources along with the archival data. The new ATCA integrated fluxes at 5.5 and $9 \mathrm{GHz}$ given in (38) have been added to the SED.

The same procedure was used to extract the ATCA flux of the source $\mathrm{K}(\log v \cdot f(v)=-14.5 \mathrm{erg}$ $\left.\mathrm{cm}^{-2} \mathrm{~s}^{-1}\right)$. The SED of the source $\mathrm{K}$ looks completely dominated by non-thermal emission without any evidence of a presence of a thermal emission in the Infrared/Optical band, showing that it is not possible to think that it is associated with any host galaxy (see Fig. 5).

The morphology of the relic image and our analysis of the SEDs of the brightest sources associated

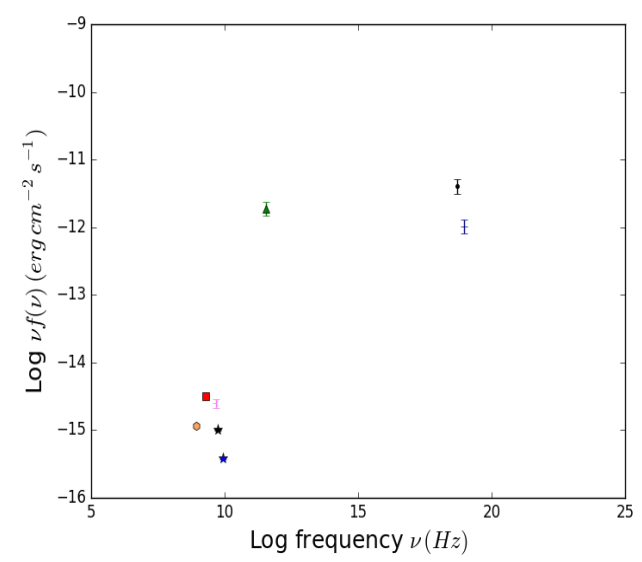

Figure 5: The SED of the source K in the ATCA radio map.

to the ATCA sources X and K suggest then that the source X can be the core of a radio galaxy and that the source $\mathrm{K}$ is its terminal radio lobe, with the remaining part of the jet visible forming a 
bridge between these two sources in the ATCA image.

In this situation, if we consider the source $\mathrm{X}$ as the core of the radio galaxy jet, a compression of the B-field (produced even by a weak shock) along the jet and lobe of this radio galaxy can revive the original electron population, thus being able to create the diffuse emission at the outskirt of the Bullet cluster referred to as the relic. The polarization and the spectral index images from $(47 ; 48)$ seem to support this conclusion. Their contour distribution in the map clearly shows that the core, the jet and the lobe are connected.

\subsection{The case of $\mathbf{A} 3667$}

Here we consider the case of the cluster A3667 where two relics which are symmetric w.r.t. the cluster centre have been observed. This phenomenology has been observed in a substantial fraction of clusters hosting relics, e.g. A1240 and A2345 (8), A3376 (4), A3667 (45), PLCKG287.0 (5), $\mathrm{ZwCl} 2341.1+0000$ (50), ZwCl 0008+52 (51), MACS J1752.0+4440 (53; 9), RXC J1314.4-2515 ((58), $0217+70(13)$.

A3667 is a southern galaxy cluster manifesting in optical as a richness class 2, Bautz-Morgan I-II intermediate type, at redshift $z=0.055(43 ; 41 ; 46)$. X-ray observations show that the cluster is

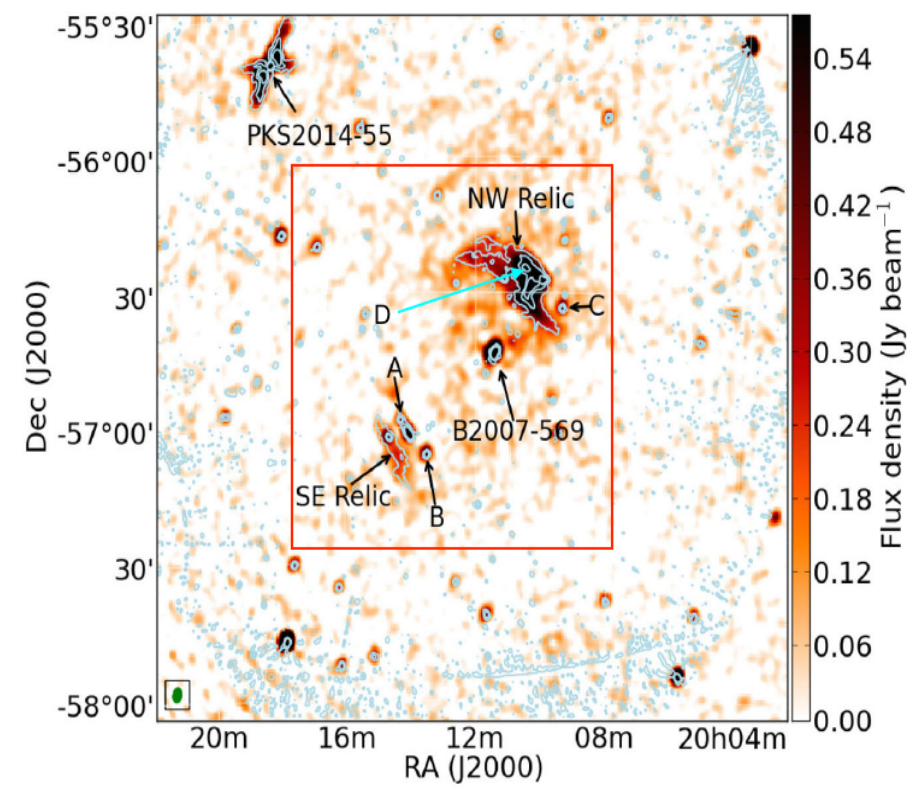

Figure 6: Radio image of the A3667 region (red box) with the radio relics and some discrete sources embedded on it. This image has been adapted from (29).

luminous $\left(L_{X}(0.4-2.4 \mathrm{keV})=5.1 \times 10^{44} \mathrm{erg} \mathrm{s}^{-1}\right)$, hot $(k T \sim 6 \mathrm{keV})$ and displays several features indicative of a major merger $(41 ; 1)$. Interferometric radio observations show the presence of two extended diffuse radio relics (labeled as NW and SE, see Fig. 6) positioned symmetrically on opposite sides of the cluster periphery with their major axis perpendicular to the direction of the cluster elongation $(41 ; 17 ; 29)$. It is important to note that it has been claimed also that this cluster contains a faint radio halo bridging with the NW relic (see, e.g., (29) for more details).

A recent XMM-Newton observation of the NW relic shows the presence of a shock (with a Mach number $\left.\mathscr{M}=2.54_{-0.43}^{+0.80}\right)(17 ; 1)$ located very close to it. Whereas, for the case of the SE relic, only 
the presence of a cold front and a possible shock is reported in the literature (see, e.g., (41)). We note that the $\mathrm{SE}$ relic might have a value $\mathscr{M}=2.4 \pm 1$ (i.e. a weak shock) from spectral index analysis (29).

The Mach number of these relics are lower than the values $\gtrsim 4$, thus suggesting an inefficient (DSA) re-acceleration of electron population in the relic area. We also note that the expected gamma-ray emission at strong shocks has not been detected. In our alternative scenario, we note that there are various radio sources in the cluster where some of the them are embedded in the relics region (see Fig. 6). Out of those ones closely related to the relics, which are labeled with A, $\mathrm{B}$ and $\mathrm{C}$, it has been suggested that the source D (RA=20 10 00; Dec=-56 21 00) might be a good candidate for the injection of electrons related to the NW relic.

We made an archival search of all sources and we only found plausible information for sources A, B and C. The source A (PMN J2014-5701) is a head-tail radio source with a bright core and narrow low surface brightness tail, with peak surface brightness $1.6 \pm 0.2 \mathrm{Jy} \mathrm{beam}^{-1}$ at $149 \mathrm{MHz}$ (29). The source B (SUMSS J201330-570552) is a suspected Fanaroff-Riley type II double-lobed radio galaxy and has a peak surface brightness of $0.9 \pm 0.1 \mathrm{Jy} \mathrm{beam}^{-1}$ at $149 \mathrm{MHz}$ (29). The source $\mathrm{C}$ is an unresolved radio galaxy (SUMSS J200926-563328) with a peak surface brightness of $1.0 \pm 0.1$ $\mathrm{Jy}_{\text {beam }}{ }^{-1}$ which is blended with the NW relic at $120 \mathrm{MHz}(29)$.

We note that for the NW relic, the source $\mathrm{C}$ or $\mathrm{D}$ might be the one providing electron population to the area, whereas for the SE relic, might be the source A or B. Thus, the seed electrons provided by them in connection with magnetic field compression due to the weak shocks can be the possible explanation for the relics appearance.

Another possibility we consider here is related to the presence of the radio galaxy MRC B2007-568; this bright radio galaxy shows an extended emission in the direction of the NW relic (see Fig.7). Interestingly, (17) noted a faint radio bridge connecting this galaxy to the western edge of the relic. Both relics seem to be approximately perpendicular to this bridge, with the SE relic (that has a lower luminosity) being located at a larger distance from the galaxy. This could suggest the possibility that the electrons are emitted by the galaxy and the resulting lobes form the radio relics when they are adiabatically compressed by the shock front.

This kind of structure, i.e. a powerful radio source embedded in a bridge connecting the centre of the cluster with a radio relic, has been observed in other clusters, e.g. the source J06587-5558 in the Bullet cluster (47), the galaxy NGC 4839 in Coma (13), and the source labeled as D in CIZA J2242.8+5301 (49). Such a coincidence suggests that this explanation could be a common possibility for the origin of radio relics.

\section{High-energy expectations}

As discussed in the Introduction, the recent upper limits set by Fermi LAT put strong limits on the level of particle acceleration in galaxy clusters, and as a consequence on the possibility that the acceleration or re-acceleration of particles from processes related to shocks is the origin of radio relics (57). The detection of the non-thermal components of galaxy clusters at high energies would be a strong information regarding the origin of non-thermal particles, and as a consequence of radio relics. However, the possibility to detect a gamma-ray emission from radio relics appears challenging. In fact, radio relics are usually located at a great distance from the clusters centers, 


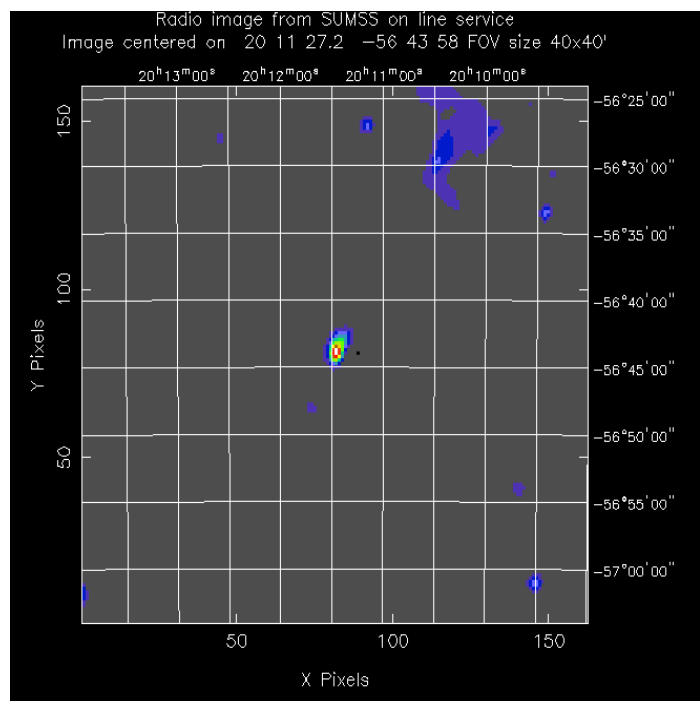

Figure 7: The SUMSS radio map centered on the radio galaxy MRC B2007-568, showing that the radio emission of the galaxy is extended in the direction of the relic (image obtained from the ASDC Sky Explorer, http://tools.asdc.asi.it/ ).

where the densities of the thermal gas and Dark Matter are supposed to be low. For example, the location of the relic in the Bullet cluster is far from both the peaks of X-ray emission and gravitational lensing maps (see, e.g., Fig. 5 in (47)), making very difficult to consider the possibility that gamma-ray emission related to hadronic interactions or Dark Matter annihilation can be detected in radio relics. We can therefore think that a high-energy emission coming from relic regions can be due to primary electrons, that, as discussed previously, is limited by the Fermi upper limits in the cluster. In principle the electrons emitting in the radio band can emit at high energies (from hard X-rays to gamma rays) by ICS of the CMB photons, whereas non-thermal bremsstrahlung is disfavoured because of the low density of thermal nuclei. Since the spectrum and the density of high-energy non-thermal electrons are strongly related to the time of their acceleration and to the distance from their acceleration source, the possibility to detect the high-E ICS emission would give a strong source of information about the origin of these electrons. In fact, if the radio relic formation is related to an adiabatic compression of electrons and magnetic fields produced in radio galaxies jets (as in our hypothesis), it is difficult to expect a high density of very-high-energy particles (with $\gamma \sim 10^{4}$ or more), because the energy losses would quickly remove these electrons from the spectrum (23), and therefore we do not expect any emission in the hard X-rays or gamma rays due to ICS, excluding possibly the regions close to the radio galaxies where the electrons were recently emitted. A possible detection of relics in these bands would instead mean that electrons with Lorentz factor $\gamma \sim 10^{4}$ are produced or accelerated far from the radio galaxies, indicating that other acceleration mechanisms must be effective.

We also note that in our hypothesis the radio jets originating the relics appear to be extended in the sky plane because they are misaligned with respect to the observer; this fact reduces relativistic beaming effects that instead can allow to detect a gamma-ray emission in less misaligned objects, like NGC 1275 in the Perseus cluster (see discussion in (20)). Finally, we note that the limited angular resolution of gamma-ray experiments will make problematic to distinguish between the 
gamma-ray emission from the centre of the cluster and from the relic. Also from this point of view, the detection of ICS emission in the hard X-ray band with high resolution instruments could provide better information.

\section{Conclusions}

In this paper we have explored the correlation between Mach numbers and the relic radio powers, the possible connection between radio relics and galaxies jets, and the possibility to test this hypothesis with high-energy observations.

We derived the $P_{1.4}-\mathscr{M}$ correlation for the sample of RRs in (29) and we found that the observed correlation is not consistent with both strong and weak shock model predictions. This brings strong problems to the commonly assumed scenario of particle (re-)acceleration at shocks through DSA for the origin of radio relics.

We discussed alternative scenarios for the correlation of relics with radio galaxy ejecta and we considered specifically the case of the relic in the Bullet cluster and the double relics in A3667 finding a good evidence of the connection between radio relics and radio galaxy jets, based on the fact that in both these clusters it is possible to identify some radio galaxies spatially located at the edge of the relic or close to it; the multi-frequency study of the different regions of the relic allows to determine which regions can be considered as the sources of the electrons, due to the presence of the optical counterpart of the radio galaxy, and which regions are instead coincident with the jets and the lobes of these radio galaxies. This connection can be explained assuming that the formation of radio relic is due to the compression (possibly operated by the weak shocks) of electrons and magnetic fields produced in radio galaxies jets. The finding that a similar structure of the location of radio relics, radio galaxies and radio bridges is observed in several clusters, can be a clue that this hypothesis is correct.

In a coming paper (Colafrancesco et al. in preparation) we will explore in more detail the correlation of RR powers and other properties of the host cluster, and we will extend the study of the connection between radio galaxies jets and radio relics to a large number of clusters thus providing more statistics to prove our hypothesis.

We finally conclude that the study of radio relics at high energies seems to be problematic, but can be possibly performed in the hard X-ray band through the ICS emission of relativistic electrons off the CMB photons. In particular, if the adiabatic compression scenario is true we expect that the ICS emission in the X-rays in the relic region (far from the radio galaxies emitting the electrons) should drop quickly at relatively low energies, whereas if other acceleration scenarios are effective the ICS can extend also to very high energies until gamma rays.

\section{Acknowledgments}

This work was supported by the Department of Science and Technology and the National Research Foundation of South Africa through a block grant to the South African Gamma-Ray Astronomy Consortium.

This work is also based on the research supported by the South African Research Chairs Initiative of the Department of Science and Technology and National Research Foundation of South Africa 
(Grant No 77948). C.M.P. and P.M. acknowledge support from the DST/NRF SKA post-graduate bursary initiative.

We thank the referee for the comments, corrections and suggestions which contributed significantly to improve the quality of the paper.

\section{References}

[1] Akamatsu, H., de Plaa, J., Kaastra, J., et al. 2012, PASJ, 64, 49

[2] Akamatsu, H., Mizuno, M., Ota, N., et al. 2017, A\&A, 600, A100

[3] Akritas, M. G., \& Bershady, M. A. 1996, ApJ, 470, 706

[4] Bagchi, J., Durret, F., Neto, G. B. L., \& Paul, S. 2006, Science, 314, 791

[5] Bagchi, J., Sirothia, S. K., Werner, N., et al. 2011, ApJL, 736, L8

[6] Basu, K., Vazza, F., Erler, J., \& Sommer, M. 2016, A\&A, 591, A142

[7] Blandford, R., \& Eichler, D. 1987, Phys. Rep., 154, 1

[8] Bonafede, A., Giovannini, G., Feretti, L., Govoni, F., \& Murgia, M. 2009, A\&A, 494, 429

[9] Bonafede, A., Brüggen, M., van Weeren, R., et al. 2012, MNRAS, 426, 40

[10] Bonafede, A., Intema, H. T., Brüggen, M., et al. 2014, ApJ, 785, 1

[11] Botteon, A., Gastaldello, F., Brunetti, G., \& Kale, R. 2016, MNRAS, 463, 1534

[12] Brienza, M., Godfrey, L., Morganti, R., et al. 2016, A\&A, 585, A29

[13] Brown, S., \& Rudnick, L. 2011, MNRAS, 412, 2

[14] Brunetti, G., Blasi, P., Cassano, R., and Gabici, S., 2004, MNRAS, 350, 1174

[15] Brunetti, G., Cassano, R., Dolag, K., \& Setti, G. 2009, A\&A, 507, 661

[16] Brunetti, G., \& Jones, T. W. 2014, International Journal of Modern Physics D, 23, 1430007-98

[17] Carretti, E., Brown, S., Staveley-Smith, L., et al. 2013, MNRAS, 430, 1414

[18] Chung, S. M., Gonzalez, A. H., Clowe, D., Markevitch, M., \& Zaritsky, D. 2010, ApJ, 725, 1536

[19] Clarke, T., Mroczkowski, T., Brown, S., et al. 2014, arXiv:1401.0329

[20] Colafrancesco, S., Marchegiani, P., \& Giommi, P. 2010, A\&A, 519, A82

[21] Donnert, J. M. F., Stroe, A., Brunetti, G., Hoang, D., \& Roettgering, H. 2016, MNRAS, 462, 2014

[22] Eckert, D., Jauzac, M., Vazza, F., et al. 2016, MNRAS, 461, 1302 
[23] Enßlin, T. A., \& Gopal-Krishna 2001, A\&A, 366, 26

[24] Feretti, L., Giovannini, G., Govoni, F., \& Murgia, M. 2012, A\&ARv, 20, 54

[25] de Gasperin, F., Ogrean, G. A., van Weeren, R. J., et al. 2015, MNRAS, 448, 2197

[26] Giacintucci, S., Venturi, T., Cassano, R., Dallacasa, D., \& Brunetti, G. 2009, ApJL, 704, L54

[27] Giovannini, G., \& Feretti, L. 2004, Journal of Korean Astronomical Society, 37, 323

[28] Griffin, R. D., Dai, X., \& Kochanek, C. S. 2014, ApJL, 795, L21

[29] Hindson, L., Johnston-Hollitt, M., Hurley-Walker, N., et al. 2014, MNRAS, 445, 330

[30] Hoeft, M., \& Brüggen, M. 2007, MNRAS, 375, 77

[31] Itahana, M., Takizawa, M., Akamatsu, H., et al. 2015, PASJ, 67, 113

[32] Johansson, D., Horellou, C., Lopez-Cruz, O., et al. 2012, A\&A, 543, A62

[33] Kale, R., Venturi, T., Giacintucci, S., et al. 2013, A\&A, 557, A99

[34] Keshet, U. 2010, arXiv:1011.0729

[35] Kierdorf, M., Beck, R., Hoeft, M., et al. 2017, A\&A, 600, A18

[36] Lakhchaura, K., \& Singh, K. P. 2014, AJ, 147, 156

[37] Liang, H., Ekers, R. D., Hunstead, R. W., Falco, E. E., \& Shaver, P. 2001, MNRAS, 328, L21

[38] Malu, S., Datta, A., \& Sandhu, P. 2016, AP\&SS, 361, 255

[39] Marchegiani, P., \& Colafrancesco, S. 2015, MNRAS, 452, 1328

[40] Miniati, F., Ryu, D., Kang, H., \& Jones, T. W., 2001, ApJ, 559, 59

[41] Owers, M. S., Couch, W. J., \& Nulsen, P. E. J. 2009, ApJ, 693, 901

[42] Petrosian, V., \& East, W. E. 2008, ApJ, 682, 175-185

[43] Rephaeli, Y., \& Gruber, D. 2004, ApJ, 606, 825

[44] Riseley, C. J., Scaife, A. M. M., Oozeer, N., Magnus, L., \& Wise, M. W. 2015, MNRAS, 447, 1895

[45] Rottgering, H. J. A., Wieringa, M. H., Hunstead, R. W., \& Ekers, R. D. 1997, MNRAS, 290, 577

[46] Sarazin, C. L., Finoguenov, A., Wik, D. R., \& Clarke, T. E. 2016, ApJ, submitted (arXiv:1606.07433)

[47] Shimwell, T. W., Brown, S., Feain, I. J., et al. 2014, MNRAS, 440, 2901 
[48] Shimwell, T. W., Markevitch, M., Brown, S., et al. 2015, MNRAS, 449, 1486

[49] Stroe, A., van Weeren, R. J., Intema, H. T., et al. 2013, A\&A, 555, A110

[50] van Weeren, R. J., Röttgering, H. J. A., Bagchi, J., et al. 2009, A\&A, 506, 1083

[51] van Weeren, R. J., Brüggen, M., Röttgering, H. J. A., \& Hoeft, M. 2011, MNRAS, 418, 230

[52] van Weeren, R. J., Brüggen, M., Röttgering, H. J. A., et al. 2011, A\&A, 533, A35

[53] van Weeren, R. J., Bonafede, A., Ebeling, H., et al. 2012, MNRAS, 425, L36

[54] van Weeren, R. J., et al., 2012, A\&A, 543, A43

[55] van Weeren, R. J., Brunetti, G., Brüggen, M., et al. 2016, ApJ, 818, 204

[56] Vazza, F., Eckert, D., Brüggen, M., \& Huber, B. 2015, MNRAS, 451, 2198

[57] Vazza, F., Brüggen, M., Wittor, D., et al. 2016, MNRAS, 459, 70

[58] Venturi, T., Giacintucci, S., Brunetti, G., et al. 2007, A\&A, 463, 937

[59] Wittor, D., Vazza, F., \& Brüggen, M. 2016, Galaxies, 4, 71

[60] Wolfe, B., \& Melia, F. 2006, ApJ, 638, 125

[61] Yuan, Z. S., Han, J. L., \& Wen, Z. L. 2015, ApJ, 813, 77 